\title{
Operation Mode Analysis of 3-RPS Parallel Manipulators based on their Design Parameters
}

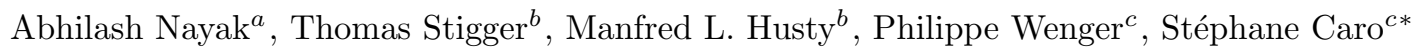 \\ ${ }^{a}$ École Centrale de Nantes, Laboratoire des Sciences du Numérique de Nantes (LS2N), 1 rue de la Noë, 44321 Nantes, \\ France, e-mail:abhilash.nayak@ls2n.fr \\ ${ }^{b}$ Unit Geometry and CAD, University of Innsbruck, Austria, e-mail: \{thomas.stigger, manfred.husty@uibk.ac.at\} \\ ${ }^{c}$ CNRS, Laboratoire des Sciences du Numérique de Nantes (LS2N), École Centrale de Nantes, 1 rue de la Noë, UMR \\ CNRS 6004, 44321 Nantes, France, e-mail: \{stephane.caro, philippe.wenger\}@ls2n.fr
}

\begin{abstract}
It is known that a 3-RPS parallel manipulator with an equilateral triangle base and an equilateral triangle platform has two operation modes [1] whereas a 3-RPS cube manipulator with a cube shaped base and an equilateral triangle platform has only one operation mode [2]. This behavior is indeed a result of the difference in the architectures of these manipulators. Therefore, this paper deals with the operation mode analysis of 3-RPS parallel manipulators based on their design parameters. Study's kinematic mapping is exploited to derive the constraint equations of the manipulators under study. A linear combination of the constraint equations independent of the joint variables is compared with a general quadric in the 7-dimensional projective space $\mathbb{P}^{7}$ to obtain some relations between the design parameters of 3-RPS manipulators with coplanar revolute joints, such that those manipulators have two operation modes. Some special cases and a numerical example are considered to emphasize the proposed approach and highlight the contributions of the paper.
\end{abstract}

Keywords: Study's kinematic mapping, 3-RPS, parallel manipulator, operation modes.

\section{Introduction}

The 3-RPS parallel manipulator is a three degree-of-freedom (DOF) spatial mechanism, initially proposed by Hunt [3]. This manipulator allows one pure vertical translation and two rotations about axes parallel to the horizontal plane, but since those axes do not remain fixed when the manipulator moves, the two

5 rotations generate two parasitic horizontal translations. The mechanism is composed of three identical limbs connecting its base to its moving platform. Each limb consists of a revolute joint, a prismatic joint and a spherical joint mounted in series.

Several arrangements of the joints are possible, e.g. the R-joint axes in the base frame can be tangential to a circle, parallel or intersect at a common point.

\footnotetext{
${ }^{*}$ Corresponding author
} 
Several research works have dealt with the kinematic analysis of the 3-RPS parallel manipulator. Huang and Fang described the constraints of the manipulator [4] using screw theory. The number of solutions to the inverse kinematics was first published by Nanua et al. [5] and Tsai [6], Self-motions [7] were investigated by Schadlbauer et al. in which a spatial 3-RPS Manipulator was considered with R-joints tangential to the base circum-circle. Workspace and joint space analysis 8 using quaternions was done by Chablat et al., and more special configurations of the 3-RPS manipulator [2] like the 3-RPS cube manipulator as well as the synthesis of design parameters with respect to specific operation modes [9] were both investigated by Nurahmi et al. Moreover, a complete algebraic analysis of the 3-RPS parallel manipulator was published, using Study's kinematic mapping in [1] and in [10. Gallardo et al. analyzed the kinematics of the 3-RPS parallel manipulator by using screw theory [1].

The motion capabilities of the 3-RPS parallel manipulator were exploited in telescope applications studied by Carretero et al. 12 and in machine tool heads, investigated by Hernandes et al. [13].

The application for medical purposes like human machine interactions were investigated in [14, including the control of the manipulator with PID controllers.

The subject of this paper is about the determination of some conditions on the design parameters of 3-RPS manipulators with coplanar revolute joint axes for those manipulators to have two operation modes.

The paper is organized as follows: Section 2 presents the architecture and parameterization of 3-RPS manipulators with coplanar revolute joint axes. Section 3 expresses their constraint equations as a function of Study parameters and independently of joint variables. Section 4 deals with the operation mode analysis of the manipulators under study and gives some conditions on their design parameters to lead to two operation modes. The operation modes of three 3-RPS parallel manipulators with coplanar revolute joints defined based on the foregoing conditions on design parameters are analyzed in Section 5 as illustrative examples. Some discussions and conclusions are given in Sec. 6.

\section{Manipulator Architectures}

The investigated spatial parallel manipulator shown in Fig. 1 consists of a moving platform connected to a fixed base with three limbs. Each limb is composed of a revolute joint, a prismatic joint and a spherical joint mounted in series 1 . The three prismatic joints are actuated. Figure 2 represents a RPS limb. The base of the 3 -RPS manipulator is specified by 3 base-points $A_{1}, A_{2}$ and $A_{3}$ in the fixed frame $\mathscr{F}_{0}$. The fixed frame is defined such that $A_{1}$ is the origin of the coordinate frame, $A_{2}$ is along the $x$-axis and $A_{3}$ is an arbitrary point in the $X Y$-plane. $B_{1}, B_{2}$ and $B_{3}$ are the vertices of the triangular moving-platform, $B_{1}$ is the origin of the moving-platform frame $\mathscr{F}_{1}, B_{2}$ is along the $x$-axis of $\mathscr{F}_{1}$ and $B_{3}$ lies in the $x y$-plane.

\footnotetext{
${ }^{1} \mathrm{~A}$ revolute, prismatic and a spherical joint are denoted by $\mathrm{R}, \mathrm{P}$ and $\mathrm{S}$ respectively.
} 


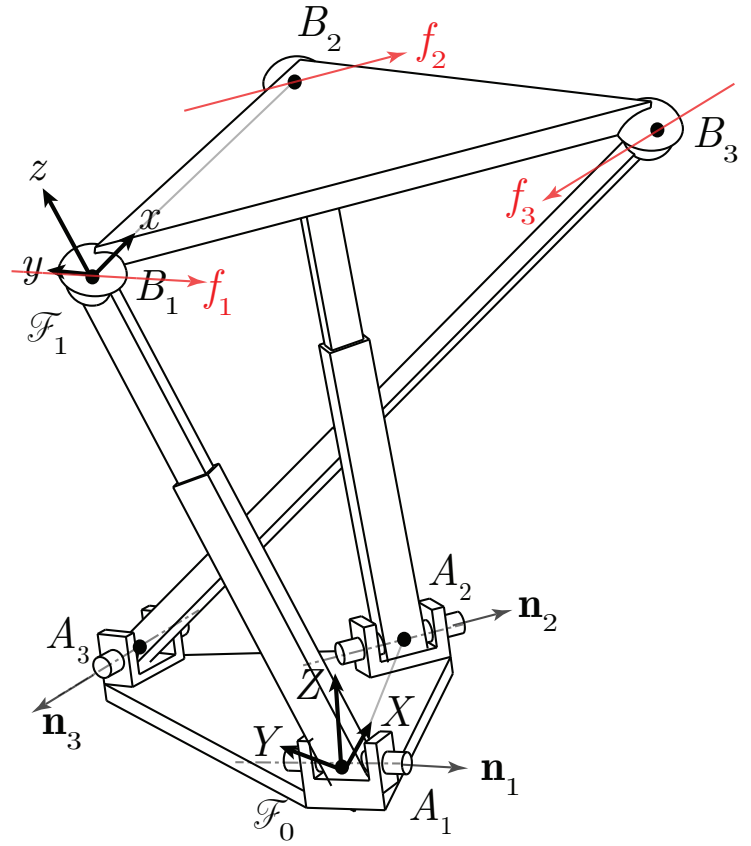

Figure 1: Architecture of a 3-RPS parallel manipulator with coplanar revolute joints

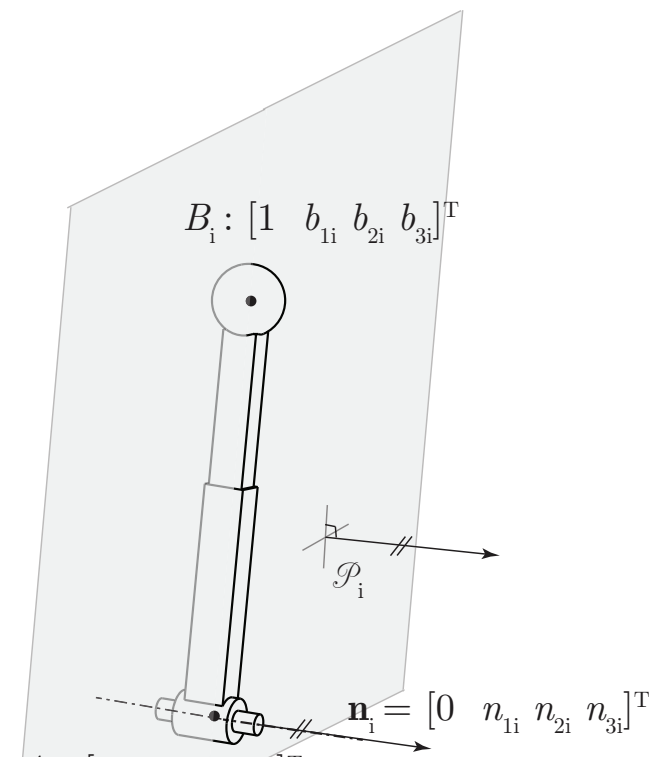

$A_{\mathrm{i}}:\left[\begin{array}{llll}1 & a_{1 \mathrm{i}} & a_{2 \mathrm{i}} & a_{3 \mathrm{i}}\end{array}\right]^{\mathrm{T}}$

Figure 2: A RPS limb

The $i$ th revolute joint axis of direction $\mathbf{n}_{i}$ is perpendicular to the direction of the $i$ th prismatic joint, namely,

$$
\mathbf{n}_{i} \cdot \overrightarrow{A_{i} B_{i}}=0, \quad i=1,2,3
$$

\section{Kinematic Modeling}

To derive the constraint equations of the 3-RPS parallel manipulators with coplanar revolute joint axes, the homogeneous coordinates of point $A_{i}$ and vector $\mathbf{n}_{i}$ are firstly expressed in frame $\mathscr{F}_{0}$ while that of the point $B_{i}$ are expressed in frame $\mathscr{F}_{1}$ :

$$
\begin{aligned}
& { }^{0} \mathbf{a}_{1}=(1,0,0,0), \quad{ }^{0} \mathbf{a}_{2}=\left(1, a_{12}, 0,0\right), \quad{ }^{0} \mathbf{a}_{3}=\left(1, a_{13}, a_{23}, 0\right), \\
& { }^{1} \mathbf{b}_{1}=(1,0,0,0), \quad{ }^{1} \mathbf{b}_{2}=\left(1, b_{12}, 0,0\right), \quad{ }^{1} \mathbf{b}_{3}=\left(1, b_{13}, b_{23}, 0\right), \\
& { }^{0} \mathbf{n}_{1}=\left(0, n_{11}, n_{21}, n_{31}\right), \quad{ }^{0} \mathbf{n}_{2}=\left(0, n_{12}, n_{22}, n_{32}\right), \quad{ }^{0} \mathbf{n}_{3}=\left(0, n_{13}, n_{23}, n_{33}\right),
\end{aligned}
$$

with the first entry of each vector being the homogenizing coordinate ${ }^{2}$. Since points $B_{i}$ are given in the moving frame, a transformation is applied to obtain it in $\mathscr{F}_{0}$ frame. Study's kinematic mapping can be used for this purpose. It is a mapping from the special Euclidean motion group $\mathrm{SE}(3)$ to a seven-dimensional projective space $\mathbb{P}^{7}\left[15\right.$. The transformation to obtain ${ }^{0} \mathbf{b}_{i}$ from ${ }^{1} \mathbf{b}_{i}$ is given in Eq. (5)

\footnotetext{
${ }^{2} \mathrm{~A}$ vector expressed in $\mathscr{F}_{0}$ is denoted as ${ }^{0}\{\cdot\}$ whereas a vector expressed in $\mathscr{F}_{1}$ is indicated as ${ }^{1}\{\cdot\}$.
} 


$$
{ }^{0} \mathbf{T}_{1}=\frac{1}{\Delta}\left[\begin{array}{cccc}
\Delta & 0 & 0 & 0 \\
d_{1} & x_{0}{ }^{2}+x_{1}{ }^{2}-x_{2}{ }^{2}-x_{3}{ }^{2} & -2 x_{0} x_{3}+2 x_{1} x_{2} & 2 x_{0} x_{2}+2 x_{1} x_{3} \\
d_{2} & 2 x_{0} x_{3}+2 x_{1} x_{2} & x_{0}{ }^{2}-x_{1}{ }^{2}+x_{2}{ }^{2}-x_{3}{ }^{2} & -2 x_{0} x_{1}+2 x_{2} x_{3} \\
d_{3} & -2 x_{0} x_{2}+2 x_{1} x_{3} & 2 x_{0} x_{1}+2 x_{2} x_{3} & x_{0}{ }^{2}-x_{1}{ }^{2}-x_{2}{ }^{2}+x_{3}{ }^{2}
\end{array}\right]
$$

with $\Delta=x_{0}^{2}+x_{1}^{2}+x_{2}^{2}+x_{3}^{2} \neq 0$

$$
\begin{aligned}
\text { and } d_{1} & =-2 x_{0} y_{1}+2 x_{1} y_{0}-2 x_{2} y_{3}+2 x_{3} y_{2}, \\
d_{2} & =-2 x_{0} y_{2}+2 x_{1} y_{3}+2 x_{2} y_{0}-2 x_{3} y_{1}, \\
d_{3} & =-2 x_{0} y_{3}-2 x_{1} y_{2}+2 x_{2} y_{1}+2 x_{3} y_{0},
\end{aligned}
$$

where $x_{j}, y_{j}, j=0,1,2,3$ are the so called Study parameters of the transformation ${ }^{0} \mathbf{T}_{1}$. A point $P=\left(x_{0}\right.$ : $\left.x_{1}: x_{2}: x_{3}: y_{0}: y_{1}: y_{2}: y_{3}\right) \in \mathbb{P}^{7}$ represents an Euclidean transformation, if and only if $P$ lies in a 6-dimensional quadric, $S_{6}^{2} \in \mathbb{P}^{7}$ called as the Study quadric:

$$
S_{6}^{2}: x_{0} y_{0}+x_{1} y_{1}+x_{2} y_{2}+x_{3} y_{3}=0
$$

The geometric constraints of the parallel manipulator can be derived as follows. As the prismatic joints are actuated, the distance between points $A_{i}$ and $B_{i}$ is equal to the prismatic joint length $r_{i}$. Therefore, the following first three distance constraints arise:

$$
g_{i}:\left({ }^{0} \mathbf{a}_{i}-{ }^{0} \mathbf{b}_{i}\right)^{T}\left({ }^{0} \mathbf{a}_{i}-{ }^{0} \mathbf{b}_{i}\right)-r_{i}^{2}=0, \quad i=1,2,3 .
$$

The next three geometric constraints of the manipulator is derived from the perpendicularity between the revolute joint and the prismatic joint direction within each limb, namely, from Eq. (1):

$$
g_{i+3}:{ }^{0} \mathbf{n}_{i}^{T}\left({ }^{0} \mathbf{a}_{i}-{ }^{0} \mathbf{b}_{i}\right)=0, \quad i=1,2,3 .
$$


As a result, the six constraint equations are expressed as follows after some mathematical simplifications:

$$
\begin{aligned}
& g_{1}:=\left(x_{0}^{2}+x_{1}^{2}+x_{2}^{2}+x_{3}^{2}\right){r_{1}}^{2}-4 y_{0}^{2}-4 y_{1}^{2}-4 y_{2}^{2}-4 y_{3}^{2}=0 \\
& g_{2}:=\left(x_{0}{ }^{2}+x_{1}{ }^{2}+x_{2}{ }^{2}+x_{3}{ }^{2}\right)\left(r_{2}^{2}-b_{12}^{2}-a_{12}{ }^{2}\right)+\left(2 x_{0}{ }^{2}+2 x_{1}{ }^{2}-2 x_{2}{ }^{2}-2 x_{3}{ }^{2}\right) a_{12} b_{12} \\
& +\left(-4 x_{0} y_{1}+4 x_{1} y_{0}-4 x_{2} y_{3}+4 x_{3} y_{2}\right) a_{12}+\left(4 x_{0} y_{1}-4 x_{1} y_{0}-4 x_{2} y_{3}+4 x_{3} y_{2}\right) b_{12} \\
& -4 y_{0}{ }^{2}-4 y_{1}{ }^{2}-4 y_{2}{ }^{2}-4 y_{3}^{2}=0 \\
& g_{3}:=\left(x_{0}{ }^{2}+x_{1}{ }^{2}+x_{2}{ }^{2}+x_{3}{ }^{2}\right)\left(r_{3}^{2}-a_{13}{ }^{2}-a_{23}^{2}-b_{13}^{2}-b_{23}^{2}\right)+\left(2 x_{0}{ }^{2}+2 x_{1}{ }^{2}-2 x_{2}{ }^{2}-2 x_{3}{ }^{2}\right) a_{13} b_{13} \\
& +\left(-4 x_{0} x_{3}+4 x_{1} x_{2}\right) a_{13} b_{23}+\left(-4 x_{0} y_{1}+4 x_{1} y_{0}-4 x_{2} y_{3}+4 x_{3} y_{2}\right) a_{13}+\left(4 x_{0} x_{3}+4 x_{1} x_{2}\right) a_{23} b_{13} \\
& +\left(2 x_{0}{ }^{2}-2 x_{1}{ }^{2}+2{x_{2}}^{2}-2 x_{3}{ }^{2}\right) a_{23} b_{23}+\left(-4 x_{0} y_{2}+4 x_{1} y_{3}+4 x_{2} y_{0}-4 x_{3} y_{1}\right) a_{23} \\
& +\left(4 x_{0} y_{1}-4 x_{1} y_{0}-4 x_{2} y_{3}+4 x_{3} y_{2}\right) b_{13}+\left(4 x_{0} y_{2}+4 x_{1} y_{3}-4 x_{2} y_{0}-4 x_{3} y_{1}\right) b_{23} \\
& -4 y_{0}^{2}-4 y_{1}^{2}-4 y_{2}^{2}-4 y_{3}^{2}=0 \\
& g_{4}:=\left(2 x_{0} y_{1}-2 x_{1} y_{0}+2 x_{2} y_{3}-2 x_{3} y_{2}\right) n_{11}+\left(2 x_{0} y_{2}-2 x_{1} y_{3}-2 x_{2} y_{0}+2 x_{3} y_{1}\right) n_{21}=0 \\
& g_{5}:=\left(2 x_{0} y_{1}-2 x_{1} y_{0}+2 x_{2} y_{3}-2 x_{3} y_{2}\right) n_{12}+\left(2 x_{0} y_{2}-2 x_{1} y_{3}-2 x_{2} y_{0}+2 x_{3} y_{1}\right) n_{22} \\
& +a_{12} n_{12}\left(x_{0}{ }^{2}+x_{1}{ }^{2}+x_{2}{ }^{2}+x_{3}{ }^{2}\right)+\left(-x_{0}{ }^{2}-x_{1}{ }^{2}+x_{2}{ }^{2}+x_{3}{ }^{2}\right) b_{12} n_{12} \\
& +\left(-2 x_{0} x_{3}-2 x_{1} x_{2}\right) b_{12} n_{22}=0 \\
& g_{6}:=\left(2 x_{0} y_{1}-2 x_{1} y_{0}+2 x_{2} y_{3}-2 x_{3} y_{2}\right) n_{13}+\left(2 x_{0} y_{2}-2 x_{1} y_{3}-2 x_{2} y_{0}+2 x_{3} y_{1}\right) n_{23} \\
& -\left(a_{13} n_{13}+a_{23} n_{23}\right)\left(x_{0}^{2}+x_{1}^{2}+x_{2}^{2}+x_{3}^{2}\right)+\left(-x_{0}{ }^{2}-x_{1}{ }^{2}+x_{2}{ }^{2}+x_{3}{ }^{2}\right) b_{13} n_{13} \\
& +\left(-x_{0}{ }^{2}+x_{1}{ }^{2}-x_{2}{ }^{2}+x_{3}{ }^{2}\right) b_{23} n_{23}+\left(-2 x_{0} x_{3}-2 x_{1} x_{2}\right) b_{13} n_{23}+\left(2 x_{0} x_{3}-2 x_{1} x_{2}\right) b_{23} n_{13}=0
\end{aligned}
$$

It should be noted that those six equations are a function of fifteen design parameters $a_{12}, a_{13}, a_{23}, b_{12}, b_{13}$, $b_{23}, n_{i j}(i, j \in\{1,2,3\})$, three actuated prismatic joint variables $r_{1}, r_{2}, r_{3}$ and the Study parameters.

\section{Operation modes}

This section aims to find the conditions on the fifteen design parameters such that the 3-RPS manipulator with coplanar revolute joints can exhibit more than one operation mode. Since the R-joint axes are assumed coplanar, $n_{31}=n_{32}=n_{33}=0$.

From the standpoint of algebraic geometry, it is known that the existence of more than one operation mode requires the factorization of a polynomial belonging to the ideal of constraint polynomials (preferably the ones independent of actuated joint variables) [1]. In this context, an ideal $\mathcal{I}$ is considered such that it is a subset of the field of Study parameters:

$$
\mathcal{I}=\left\langle g_{4}, g_{5}, g_{6}\right\rangle \mid \mathcal{I} \subseteq K\left[x_{0}, x_{1}, x_{2}, x_{3}, y_{0}, y_{1}, y_{2}, y_{3}\right]
$$


From the definition of an ideal, if a polynomial $g \in \mathcal{I}$ and $h \in K, K$ being the field over which the ideal $\mathcal{I}$ is defined, then $h g \in \mathcal{I}$ [16. From Eqs. (9) and (10), a polynomial $g$ is defined such that

$$
g=h_{1} g_{4}+h_{2} g_{5}+h_{3} g_{6} \in \mathcal{I}, \text { where } h_{i} \neq 0 \in K\left[x_{0}, x_{1}, x_{2}, x_{3}, y_{0}, y_{1}, y_{2}, y_{3}\right], i=1,2,3
$$

For simplicity, $h_{i}$ is only allowed to be a function of design parameters. This assumption forces the polynomial $g$ to be quadratic. To this end, the problem boils down to find the coefficients $h_{i}$ such that $g$ can be factorized. In search of linear factors, two general linear equations are introduced in Eq. (12) and are multiplied to obtain a general quadratic polynomial $s_{12}$ in the kinematic image space, $\mathbb{P}^{7}$.

$$
\begin{aligned}
& s_{1}: m_{1} x_{0}+m_{2} x_{1}+m_{3} x_{2}+m_{4} x_{3}+m_{5} y_{0}+m_{6} y_{1}+m_{7} y_{2}+m_{8} y_{3}=0 \\
& s_{2}: n_{1} x_{0}+n_{2} x_{1}+n_{3} x_{2}+n_{4} x_{3}+n_{5} y_{0}+n_{6} y_{1}+n_{7} y_{2}+n_{8} y_{3}=0 \\
& s_{12}=s_{1} \cdot s_{2}
\end{aligned}
$$

where $m_{k}$ and $n_{k}, k=1, \ldots, 8$ are constants. Equating the respective coefficients of $g$ and $s_{12}$ leads to a system of 36 linear equations in 31 unknowns. Solving for all the parameters $b_{12}, b_{13}, b_{23}, a_{12}, a_{13}, a_{23}$, $h_{1}, h_{2}, h_{3}, m_{k}, n_{k}, k=1, \ldots, 8$, yields 36 solutions. It is noteworthy that some equations are dependent and the system is underdetermined. Nonetheless, the solve function in Maple parametrizes the solutions in terms of one or more unknowns. Investigating the solution set reveals that there are some trivial solutions (complex ones and the ones with $h_{i}=0, a_{12}=0, a_{13}=0, a_{23}=0, b_{12}=0, b_{13}=0$ or $\left.b_{23}=0\right)$ and some are special cases of the general one $\left(n_{i j}=0, i=1,2, j=1,2,3\right)$. The focus is on the two general solutions:

$\underline{\text { Solution } 1}$

$$
\begin{aligned}
& m_{1}=\frac{\left(n_{13} \pm \sqrt{n_{13}^{2}+n_{23}^{2}}\right) m_{4}}{n_{23}}, m_{2}=0, m_{3}=0, m_{4}=m_{4}, m_{5}=0, m_{6}=0, m_{7}=0, m_{8}=0, \quad(13 \mathrm{a}) \\
& n_{1}=\frac{2\left(n_{13} \pm \sqrt{n_{13}{ }^{2}+n_{23}}\right) b_{23} h_{3}}{m_{4}}, n_{2}=0, n_{3}=0, n_{4}=2 \frac{b_{23} h_{3} n_{23}}{m_{4}}, n_{5}=0, n_{6}=0, n_{7}=0, n_{8}=0 \\
& a_{12}=\frac{\left(n_{11} n_{22}-n_{12} n_{21}\right)\left(a_{13} n_{13}+a_{23} n_{23}\right)}{\left(n_{11} n_{23}-n_{13} n_{21}\right) n_{12}}, a_{13}=a_{13}, a_{23}=a_{23} \\
& b_{12}=-\frac{b_{23}\left(n_{13}^{2}+n_{23}^{2}\right)\left(n_{11} n_{22}-n_{12} n_{21}\right)}{\left(n_{12} n_{23}-n_{13} n_{22}\right)\left(n_{11} n_{23}-n_{13} n_{21}\right)}, b_{13}=-\frac{b_{23}\left(n_{12} n_{13}+n_{22} n_{23}\right)}{n_{12} n_{23}-n_{13} n_{22}}, b_{23}=b_{23} \\
& h_{1}=\frac{h_{3}\left(n_{12} n_{23}-n_{13} n_{22}\right)}{n_{11} n_{22}-n_{12} n_{21}}, h_{2}=-\frac{h_{3}\left(n_{11} n_{23}-n_{13} n_{21}\right)}{n_{11} n_{22}-n_{12} n_{21}}, h_{3}=h_{3}
\end{aligned}
$$


Solution 2

$$
\begin{aligned}
& m_{1}=0, m_{2}=\frac{\left(n_{13} \pm \sqrt{n_{13}{ }^{2}+n_{23}{ }^{2}}\right) m_{3}}{n_{23}}, m_{3}=m_{3}, m_{4}=0, m_{5}=0, m_{6}=0, m_{7}=0, m_{8}=0, \\
& n_{1}=0, n_{2}=\frac{2 b_{23} h_{3} n_{23}^{2}}{\left(n_{13} \pm \sqrt{n_{13}{ }^{2}+n_{23}{ }^{2}}\right) m_{3}}, n_{3}=-2 \frac{b_{23} h_{3} n_{23}}{m_{3}}, n_{4}=0, n_{5}=0, n_{6}=0, n_{7}=0, n_{8}=0 \\
& a_{12}=\frac{\left(n_{11} n_{22}-n_{12} n_{21}\right)\left(a_{13} n_{13}+a_{23} n_{23}\right)}{\left(n_{11} n_{23}-n_{13} n_{21}\right) n_{12}}, a_{13}=a_{13}, a_{23}=a_{23} \\
& b_{12}=\frac{b_{23}\left(n_{13}{ }^{2}+n_{23}{ }^{2}\right)\left(n_{11} n_{22}-n_{12} n_{21}\right)}{\left(n_{12} n_{23}-n_{13} n_{22}\right)\left(n_{11} n_{23}-n_{13} n_{21}\right)}, b_{13}=\frac{b_{23}\left(n_{12} n_{13}+n_{22} n_{23}\right)}{n_{12} n_{23}-n_{13} n_{22}}, b_{23}=b_{23} \\
& h_{1}=\frac{h_{3}\left(n_{12} n_{23}-n_{13} n_{22}\right)}{n_{11} n_{22}-n_{12} n_{21}}, h_{2}=-\frac{h_{3}\left(n_{11} n_{23}-n_{13} n_{21}\right)}{n_{11} n_{22}-n_{12} n_{21}}, h_{3}=h_{3}
\end{aligned}
$$

Upon substitution of Solution 1 into Eq. (11) or Eq. 12 that describe the general quadric, the following conic comes out:

$$
s_{12}=g=2 b_{23} h_{3}\left(2 n_{13} x_{0} x_{3}-n_{23} x_{0}^{2}+n_{23} x_{3}^{2}\right)
$$

The conic is degenerate and can be factorized as follows:

$$
\begin{aligned}
s_{12}=g & =2 \frac{b_{23} h_{3}}{n_{23}}\left(x_{3} \sqrt{n_{13}^{2}+n_{23}^{2}}+n_{13} x_{3}-n_{23} x_{0}\right)\left(x_{3} \sqrt{n_{13}^{2}+n_{23}^{2}}-n_{13} x_{3}+n_{23} x_{0}\right), n_{23} \neq 0 \\
& =-2 b_{23} h_{3} n_{23}\left(x_{0}-x_{3}\left(\sqrt{\hat{n}^{2}+1}+\hat{n}\right)\right)\left(x_{0}+x_{3}\left(\sqrt{\hat{n}^{2}+1}-\hat{n}\right)\right), \hat{n}=\frac{n_{13}}{n_{23}}, n_{23} \neq 0 .
\end{aligned}
$$

bringing to light two operation modes characterized by:

$$
\begin{aligned}
& \text { Operation mode } 1: x_{0}-x_{3}\left(\sqrt{\hat{n}^{2}+1}+\hat{n}\right)=0 \\
& \text { Operation mode } 2: x_{0}+x_{3}\left(\sqrt{\hat{n}^{2}+1}-\hat{n}\right)=0, \quad \hat{n}=\frac{n_{13}}{n_{23}}, n_{23} \neq 0 .
\end{aligned}
$$

In the same vein, upon substituting Solution 2 into Eq. (11) or Eq. (12) results in the general quadric

$$
s_{12}=g=-2\left(2 n_{13} x_{1} x_{2}-n_{23} x_{1}^{2}+n_{23} x_{2}^{2}\right) b_{23} h_{3},
$$

which splits into two polynomials characterizing the following two operation modes:

$$
\begin{aligned}
& \text { Operation mode } 1: x_{1}-x_{2}\left(\sqrt{\hat{n}^{2}+1}+\hat{n}\right)=0 \\
& \text { Operation mode } 2: x_{1}+x_{2}\left(\sqrt{\hat{n}^{2}+1}-\hat{n}\right)=0, \quad \hat{n}=\frac{n_{13}}{n_{23}}, n_{23} \neq 0
\end{aligned}
$$

As a result, when the design parameters $b_{12}, b_{13}$ and $b_{23}$ follow the ratio

$$
\begin{aligned}
b_{12}: b_{13}: b_{23}= & \left(n_{11} n_{22}-n_{12} n_{21}\right)\left(n_{13}^{2}+n_{23}^{2}\right) \\
& :\left(n_{12} n_{13}+n_{22} n_{23}\right)\left(-n_{13} n_{21}+n_{11} n_{23}\right) \\
& : \pm\left(-n_{13} n_{22}+n_{12} n_{23}\right)\left(-n_{13} n_{21}+n_{11} n_{23}\right),
\end{aligned}
$$


and parameters $a_{12}, a_{13}$ and $a_{23}$ satisfy the relation

$$
\left(n_{11} n_{23}-n_{13} n_{21}\right) a_{12} n_{12}+\left(n_{12} n_{21}-n_{11} n_{22}\right)\left(+a_{13} n_{13}+a_{23} n_{23}\right)=0,
$$

then, the 3-RPS parallel manipulator with coplanar revolute joint axes has two operation modes characterized by Eqs. (18) or 20). To derive these characteristic polynomials starting from the plane constraints, the scalar coefficients of their linear combination must follow the ratio

$$
h_{1}: h_{2}: h_{3}=n_{12} n_{23}-n_{13} n_{22}: n_{11} n_{23}-n_{13} n_{21}: n_{11} n_{22}-n_{12} n_{21}
$$

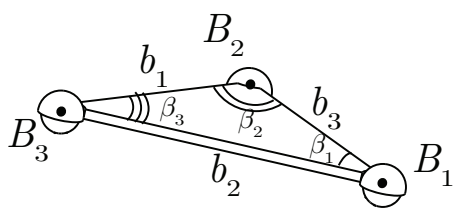

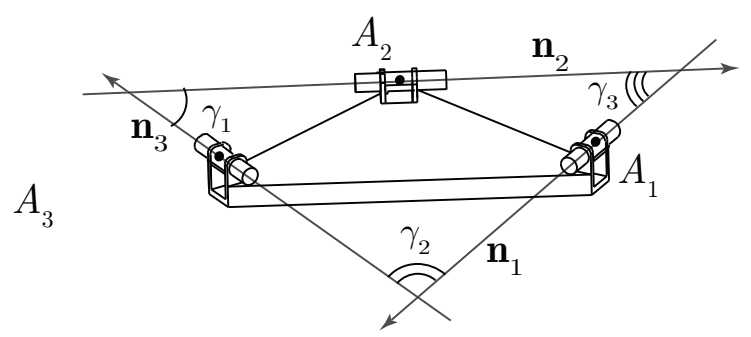

Figure 3: Similarity condition between the moving platform triangle and the triangle enclosed by the $\mathrm{R}$-joint axes.

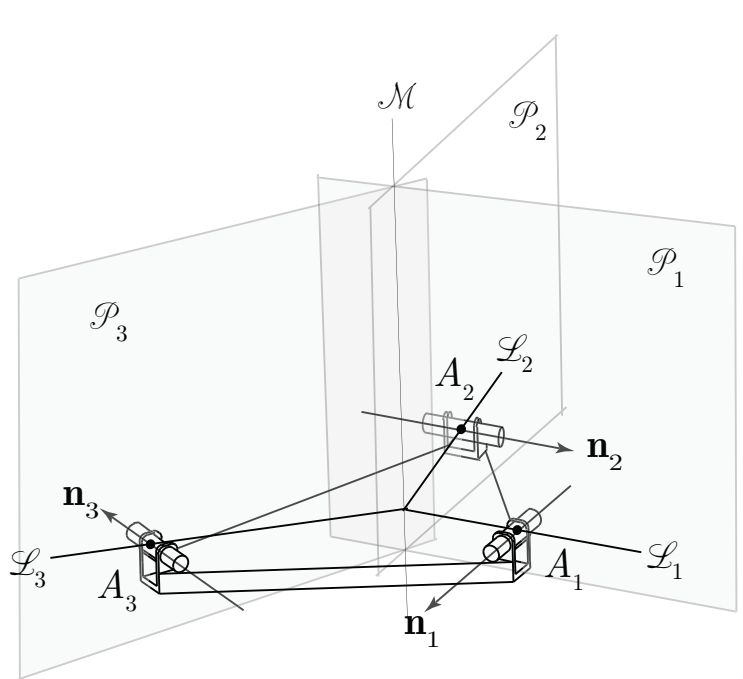

Figure 4: The planes normal to R-joint axes must have a common line of intersection.

The condition in Eq. 21) can be geometrically interpreted as the similarity (also called as homothety) between the moving platform triangle and the triangle enclosed by the three R-joint axes. This claim is proven as follows. Figure 3 shows the moving platform triangle and the triangle enclosed by the revolute joints. The sides of the moving platform triangle are $b_{1}=\sqrt{\left(b_{13}-b_{12}\right)^{2}+b_{23}^{2}}, b_{2}=\left|b_{13}-b_{12}\right|$ and $b_{3}=\left|b_{12}\right|$. Knowing the sides, the cosine of the angles $\beta_{1}, \beta_{2}$ and $\beta_{3}$ can be determined using the cosine rule. Similarly, the cosine of the angles $\gamma_{1}, \gamma_{2}$ and $\gamma_{3}$ between the R-joint axes $\mathbf{n}_{1}, \mathbf{n}_{2}$ and $\mathbf{n}_{3}$, can be determined. Equating the cosine of respective angles results in three equations ${ }^{3}$.

$$
\cos \left(\beta_{i}\right)=\cos \left(\gamma_{i}\right) \Longrightarrow \frac{b_{j}^{2}+b_{k}^{2}-b_{i}^{2}}{2 b_{j} b_{k}}=\frac{\mathbf{n}_{j} \mathbf{n}_{k}}{\left\|\mathbf{n}_{j}\right\|\left\|\mathbf{n}_{k}\right\|} \quad i, j, k=(123)
$$

\footnotetext{
${ }^{3}$ The notation $(1,2,3)$ represents a cyclic permutation. It means that $i, j, k$ are initially assigned to values $1,2,3$, respectively, $2,3,1$, subsequently and $3,1,2$, finally.
} 
Solving the equations for $b_{12}, b_{13}$ and $b_{23}$ yields the conditions in Eq. 21) proving that the considered triangles are similar (or homothetic).

Besides, Eq. 222 has a geometrical meaning too. It can be written as the determinant of a matrix, $\mathbf{P}$ :

$$
|\mathbf{P}|=\left|\begin{array}{ccc}
n_{11} & n_{21} & 0 \\
n_{12} & n_{22} & -n_{12} a_{12} \\
n_{13} & n_{23} & -n_{13} a_{13}-n_{23} a_{23}
\end{array}\right|=0
$$

$|\mathbf{P}|$ is the Grassmannian of three lines $\mathscr{L}_{1}, \mathscr{L}_{2}$ and $\mathscr{L}_{3}$ which are the projections of planes $\mathscr{P}_{1}, \mathscr{P}_{2}$ and $\mathscr{P}_{3}$ onto the $X Y$-plane as shown in Fig. 4. The equation of a line $\mathscr{L}_{i}, i=1,2,3$, orthogonal to R-joint axis $\mathbf{n}_{i}$ and passing through a point $A_{i}$ is $n_{1 i} x_{0}+n_{2 i} y_{0}-\mathbf{n}_{i}^{T} \mathbf{a}_{i}, i=1,2,3$. Therefore, $|\mathbf{P}|=0$ implies that lines $\mathscr{L}_{i}$ are concurrent, namely, planes $\mathscr{P}_{1}, \mathscr{P}_{2}$ and $\mathscr{P}_{3}$ intersect at line $\mathscr{M}$.

Thus, a 3-RPS parallel manipulator with coplanar revolute joint axes will have two operation modes if the following geometric conditions are satisfied

i Moving platform triangle is homothetic to the triangle enclosed by the revolute joint axes.

ii The three planes normal to the three revolute joint axes, respectively, have a common line of intersection. It can be shown that the above conditions are also necessary for the existence of two operation modes as explained thereafter.

Equations $(18)$ and 20 are the polynomials characterizing the two operation modes. The transition between them is when both polynomials vanish at the same time, i.e., when $x_{0}=x_{3}=0$ for Solution 1 and when $x_{1}=x_{2}=0$ for Solution 2. Transition pose is known to be a constraint singularity and in case of Solution 1, it corresponds to configurations in which the moving platform is upside down as explained in 11, 10. For Solution 2, the transition pose corresponds to configurations in which the moving platform is in an upright position parallel to the fixed base. The existence of two operation modes definitely implies a constraint singularity that separates those operation modes but the reciprocity is not necessarily true as explained in subsection 5.2. This fact is exploited to prove the necessary conditions for a 3-RPS PM to have two operation modes.

According to screw theory, it is well known that a parallel manipulator reaches a constraint singularity when its constraint wrench system is rank deficient [17, 18. At this instant, the PM gains at least one dof. The constraint wrench system of a 3-RPS PM shown in Fig. 1 is spanned by three forces $f_{1}, f_{2}$ and $f_{3}$ that are parallel to the revolute joint axes $\mathbf{n}_{1}, \mathbf{n}_{2}$ and $\mathbf{n}_{3}$ and pass through points $B_{1}, B_{2}$ and $B_{3}$, respectively. A constraint singularity implies that variety spanned by these three lines has a rank lower than 3 . This can happen only when these lines reduce to a planar pencil of lines, i.e., when they are coplanar and concurrent. 
The Plücker coordinates of the force lines can be written as follows:

$$
\begin{aligned}
& \mathbf{f}_{1}=\left[{ }^{0} \mathbf{n}_{1},{ }^{0} \mathbf{b}_{1} \times{ }^{0} \mathbf{n}_{1}\right] \\
& \mathbf{f}_{2}=\left[{ }^{0} \mathbf{n}_{2},{ }^{0} \mathbf{b}_{2} \times{ }^{0} \mathbf{n}_{2}\right] \\
& \mathbf{f}_{3}=\left[{ }^{0} \mathbf{n}_{3},{ }^{0} \mathbf{b}_{3} \times{ }^{0} \mathbf{n}_{3}\right]
\end{aligned}
$$

\section{Coplanarity condition}

Any two lines intersect when the reciprocal product of their Plücker coordinates vanishes. Therefore, the coplanarity condition can be formulated as the mutual vanishing of the reciprocal product between the force lines yielding the following three equations:

$$
\begin{aligned}
& E_{1}:=2 \frac{b_{12}\left(x_{0} x_{2}-x_{1} x_{3}\right)\left(n_{11} n_{22}-n_{12} n_{21}\right)}{x_{0}^{2}+x_{1}^{2}+x_{2}^{2}+x_{3}^{2}}=0 \\
& E_{2}:=2 \frac{\left(n_{11} n_{23}-n_{13} n_{21}\right)\left(b_{13} x_{0} x_{2}-b_{13} x_{1} x_{3}-b_{23} x_{0} x_{1}-b_{23} x_{2} x_{3}\right)}{x_{0}^{2}+x_{1}^{2}+x_{2}{ }^{2}+x_{3}{ }^{2}}=0 \\
& E_{3}:-2 \frac{\left(n_{12} n_{23}-n_{13} n_{22}\right)\left(b_{12} x_{0} x_{2}-b_{12} x_{1} x_{3}-b_{13} x_{0} x_{2}+b_{13} x_{1} x_{3}+b_{23} x_{0} x_{1}+b_{23} x_{2} x_{3}\right)}{x_{0}{ }^{2}+x_{1}{ }^{2}+x_{2}{ }^{2}+x_{3}{ }^{2}}=0
\end{aligned}
$$

Solving the previous system of equations in Eq. (27) for Study parameters $x_{0}, x_{1}, x_{2}, x_{3}$ gives two solutions:

$$
\begin{aligned}
& x_{0}=x_{3}=0, \\
& x_{1}=x_{2}=0 .
\end{aligned}
$$

Calculating ${ }^{0} \mathbf{b}_{i}, i=1,2,3$ with solutions 28 or 29 shows that the $z$-coordinates of the resulting points are the same proving that they indeed lie in a plane parallel to the $X Y$-plane.

\section{Concurrency condition}

Case 1: $x_{0}=x_{3}=0$

The $z$-coordinate of points $B_{i}$ is expressed $\frac{-2\left(x_{1} y_{2}-x_{2} y_{1}\right)}{x_{1}^{2}+x_{2}^{2}}$. Without loss of generality, these lines can now be projected to the $X Y$-plane to simplify the concurrency condition. Their projections have the equations:

$$
L_{i}:=-n_{2 i} X+n_{1 i} Y-\left(-n_{2 i}{ }^{0} b_{i x}+n_{1 i}{ }^{0} b_{i y}\right)=0, i=1,2,3,
$$

where ${ }^{0} b_{i y}$ and ${ }^{0} b_{i y}$ are the $x$ - and $y$-coordinates of point $B_{i}$, respectively. Therefore, the condition for 
concurrency of the three lines defined by equations $L_{i}=0$ is

$$
\begin{aligned}
\left|\begin{array}{lll}
-n_{21} & n_{11} & n_{21}{ }^{0} b_{1 x}-n_{11}{ }^{0} b_{1 y} \\
-n_{22} & n_{12} & n_{22}{ }^{0} b_{2 x}-n_{12}{ }^{0} b_{2 y} \\
-n_{23} & n_{13} & n_{23}{ }^{0} b_{3 x}-n_{13}{ }^{0} b_{3 y}
\end{array}\right| \\
=\left(b_{12} n_{11} n_{22} n_{23}-b_{12} n_{13} n_{21} n_{22}-b_{13} n_{11} n_{22} n_{23}+b_{13} n_{12} n_{21} n_{23}-b_{23} n_{11} n_{13} n_{22}+b_{23} n_{12} n_{13} n_{21}\right)\left(x_{2}^{2}-x_{1}^{2}\right) \\
\quad+2\left(b_{12} n_{11} n_{12} n_{23}-b_{12} n_{12} n_{13} n_{21}-b_{13} n_{11} n_{13} n_{22}+b_{13} n_{12} n_{13} n_{21}+b_{23} n_{11} n_{22} n_{23}-b_{23} n_{12} n_{21} n_{23}\right) x_{1} x_{2} \\
=0
\end{aligned}
$$

Equating the coefficients to zero leads to the following relations between the design parameters:

$$
b_{12}=-\frac{\left(n_{11} n_{22}-n_{12} n_{21}\right) b_{23}\left(n_{13}{ }^{2}+n_{23}{ }^{2}\right)}{\left(-n_{13} n_{22}+n_{12} n_{23}\right)\left(-n_{13} n_{21}+n_{11} n_{23}\right)}, \quad b_{13}=-\frac{\left(n_{12} n_{13}+n_{22} n_{23}\right) b_{23}}{n_{12} n_{23}-n_{13} n_{22}}, \quad b_{23}=b_{23}
$$

which are exactly those defined by Eq. 13d).

Furthermore, upon substitution of the values of $b_{12}$ and $b_{13}$ and $x_{0}=x_{3}=0$ in constraint equations $g_{1}=g_{2}=g_{3}=0$ defined by Eqs. 9d)- (9f) and eliminating $b_{23}$, results in the following equation:

$$
\left(n_{11} n_{23}-n_{13} n_{21}\right) a_{12} n_{12}+\left(n_{12} n_{21}-n_{11} n_{22}\right)\left(+a_{13} n_{13}+a_{23} n_{23}\right)=0,
$$

which is the relation derived in Eq. 222].

Case 2: $x_{1}=x_{2}=0$

In this case, we obtain the following symmetric relations between the design parameters:

$$
\begin{aligned}
& b_{12}=\frac{\left(n_{11} n_{22}-n_{12} n_{21}\right) b_{23}\left(n_{13}^{2}+n_{23}^{2}\right)}{\left(-n_{13} n_{22}+n_{12} n_{23}\right)\left(-n_{13} n_{21}+n_{11} n_{23}\right)}, \quad b_{13}=\frac{\left(n_{12} n_{13}+n_{22} n_{23}\right) b_{23}}{n_{12} n_{23}-n_{13} n_{22}}, \quad b_{23}=b_{23}, \\
& \left(n_{11} n_{23}-n_{13} n_{21}\right) a_{12} n_{12}+\left(n_{12} n_{21}-n_{11} n_{22}\right)\left(+a_{13} n_{13}+a_{23} n_{23}\right)=0,
\end{aligned}
$$

that corresponds to the relations derived in Eqs. $14 \mathrm{~d}$ and 22 , respectively.

As a conclusion, the following theorem can be stated:

Theorem 1 A 3-RPS parallel manipulator with coplanar revolute joint axes will have two operation modes if and only if the following geometric conditions are satisfied

i Moving platform triangle is homothetic to the triangle enclosed by the revolute joint axes.

ii The three planes normal to the three revolute joint axes, respectively, have a common line of intersection.

Since the relationship between the number of operation modes and architecture is established, the design parameters can be chosen in such a way that a constraint singularity is avoided. 
In case the revolute joint axes are no longer coplanar, equating a general quadric in $\mathbb{P}^{7}$ defined in Eq. 12 with the linear combination of the plane constraint polynomials $g_{4}, g_{5}$ and $g_{6}$ shown in Eqs. (9d)-(9f), does not yield any solution. The problem of finding the influence of design parameters on the operation modes of a general 3-RPS parallel manipulator is left for future work.

\section{Examples}

In this section, some example 3-RPS manipulators are considered to verify the proposed conditions.

5.1. Example 1: 3-RPS parallel manipulator with $n_{23}=0$

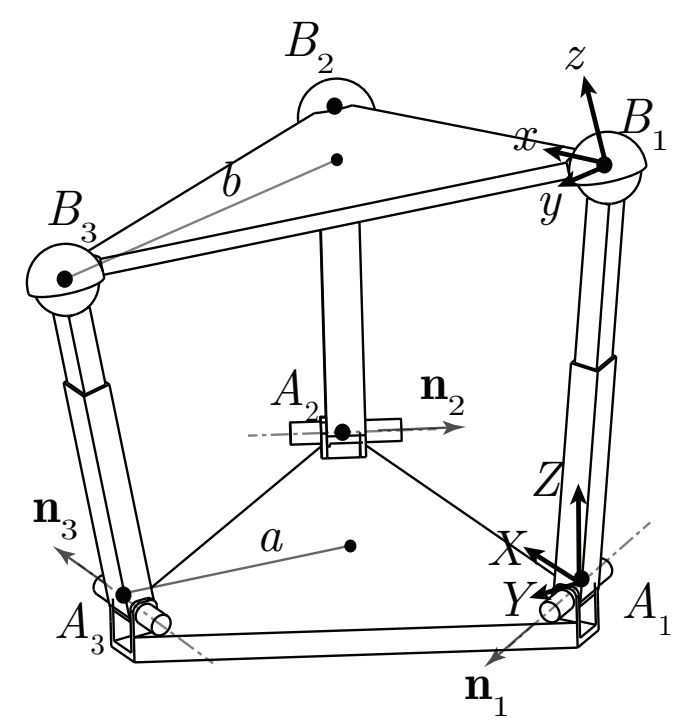

Figure 5: A 3-RPS parallel manipulator with $n_{23}=0$

The well-known 3-RPS parallel manipulator introduced by Hunt 3 has been the spotlight of numerous research topics and applications [4, 1, 8, 2, 1, 10, 11, 14. Its architecture is shown in Fig. 5. The moving platform and the base are equilateral triangles with circum-radius $b$ and $a$, respectively. The R-joint axes are coplanar and tangential to the base circum-circle. The P-joint in each leg is normal to its corresponding R-joint axis. The design parameters are listed below:

$$
\begin{aligned}
& a_{12}=\sqrt{3} a, \quad a_{13}=\frac{\sqrt{3}}{2} a, \quad a_{23}=\frac{3}{2} a, \\
& b_{12}=\sqrt{3} b, \quad b_{13}=\frac{\sqrt{3}}{2} b, \quad b_{23}=\frac{3}{2} b, \\
& n_{11}=\frac{1}{2}, \quad n_{21}=-\frac{\sqrt{3}}{2}, \quad n_{31}=0, \quad n_{12}=\frac{1}{2}, \quad n_{22}=\frac{\sqrt{3}}{2}, \quad n_{32}=0, \quad n_{13}=-1, \quad n_{23}=0, \quad n_{33}=0 .
\end{aligned}
$$


Calculating the right hand side of Eq. 211) gives $b_{12}: b_{13}: b_{23}=\frac{\sqrt{3}}{2}: \frac{\sqrt{3}}{4}: \pm \frac{3}{4}$, which is consistent with the design parameters listed in Eq. [36]. It is also straightforward to see that the design parameters satisfy Eq. 222. Thus, according to Theorem 11, the manipulator must exhibit two operation modes. To determine the characteristic equations of the operation modes, the plane constraint equations corresponding to Eq. (8) are considered:

$$
\begin{aligned}
g_{4}:= & -\sqrt{3} x_{0} y_{2}+\sqrt{3} x_{1} y_{3}+\sqrt{3} x_{2} y_{0}-\sqrt{3} x_{3} y_{1}+x_{0} y_{1}-x_{1} y_{0}+x_{2} y_{3}-x_{3} y_{2}=0 \\
g_{5}:= & \sqrt{3} x_{0} y_{2}-\sqrt{3} x_{1} y_{3}-\sqrt{3} x_{2} y_{0}+\sqrt{3} x_{3} y_{1}+x_{0} y_{1}-x_{1} y_{0}+x_{2} y_{3}-x_{3} y_{2}+\frac{\sqrt{3}}{2}\left(x_{0}{ }^{2}+x_{1}{ }^{2}+x_{2}{ }^{2}\right. \\
& \left.+x_{3}{ }^{2}\right) a-\frac{\sqrt{3}}{2}\left(2 \sqrt{3} x_{0} x_{3}+2 \sqrt{3} x_{1} x_{2}+x_{0}{ }^{2}+x_{1}{ }^{2}-x_{2}{ }^{2}-x_{3}{ }^{2}\right) b=0 \\
g_{6}:= & -2 x_{0} y_{1}+2 x_{1} y_{0}-2 x_{2} y_{3}+2 x_{3} y_{2}-\frac{\sqrt{3}}{2}\left(x_{0}{ }^{2}+x_{1}{ }^{2}+x_{2}{ }^{2}+x_{3}{ }^{2}\right) a-\frac{\sqrt{3}}{2}\left(2 \sqrt{3} x_{0} x_{3}-2 \sqrt{3} x_{1} x_{2}\right. \\
& \left.-x_{0}{ }^{2}-x_{1}{ }^{2}+x_{2}{ }^{2}+x_{3}{ }^{2}\right) b=0 .
\end{aligned}
$$

Equation 233 can be used to find the constants $h_{1}, h_{2}$ in terms of $h_{3}$ to be multiplied to the constraint polynomials $g_{4}, g_{5}$ and $g_{6}$, respectively to obtain a factorable polynomial $g$. For this manipulator, the design parameters yield $h_{1}=h_{3}$ and $h_{2}=h_{3}$. Thus from Eqs. (11) and (37)

$$
g=h_{1} g_{4}+h_{2} g_{5}+h_{3} g_{6}=h_{3}\left(g_{4}+g_{5}+g_{6}\right)=-6 h_{3} b x_{0} x_{3}
$$

showing that the manipulator at hand can have two operation modes characterized by $x_{0}=0$ and $x_{3}=0$ as already presented in [1, 10. In fact, substituting $n_{23}=0$ in Eq. 15) results in the factorable polynomial $x_{0} x_{3}$.

5.2. Example 2: 3-RPS parallel manipulator with $n_{13}=0$

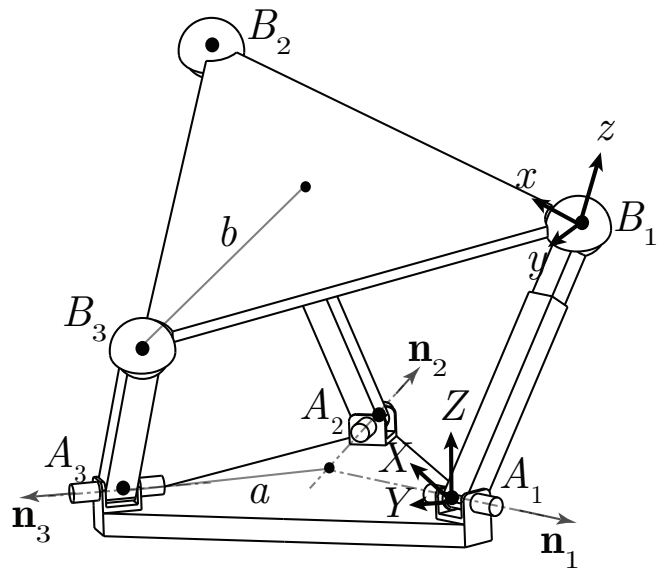

Figure 6: A 3-RPS parallel manipulator with $n_{13}=0$ 
Another special case of the 3-RPS parallel manipulator is when the R-joint axes are intersecting as shown in Fig. 6. The base and the platform are still equilateral triangles with circum-radius $a$ and $b$, respectively. Also, the P-joints are orthogonal to their corresponding R-joint axes in each leg. The design parameters for this manipulator are listed below:

$$
\begin{aligned}
& a_{12}=\sqrt{3} a, \quad a_{13}=\frac{\sqrt{3}}{2} a, \quad a_{23}=\frac{3}{2} a \\
& b_{12}=\sqrt{3} b, \quad b_{13}=\frac{\sqrt{3}}{2} b, \quad b_{23}=\frac{3}{2} b \\
& n_{11}=\frac{\sqrt{3}}{2}, \quad n_{21}=\frac{1}{2}, \quad n_{31}=0, \quad n_{12}=\frac{\sqrt{3}}{2}, \quad n_{22}=-\frac{1}{2}, \quad n_{32}=0, \quad n_{13}=0, \quad n_{23}=1, \quad n_{33}=0
\end{aligned}
$$

The ratio between $b_{12}, b_{13}$ and $b_{23}$ calculated using Eq. 21) gives $b_{12}: b_{13}: b_{23}=-\frac{\sqrt{3}}{2}:-\frac{\sqrt{3}}{4}: \pm \frac{3}{4}$, which is consistent with the design parameters listed in Eq. 41]. Thus, the condition i. of Theorem 1 is satisfied. It can also be verified by the fact that the R-joint axes intersect in a point, which is homothetic with 90 the moving platform equilateral triangle. On the other hand, the left hand side of Eq. $(22)$ gives $\frac{-3 \sqrt{3} a}{2} \neq 0$ proving that this manipulator can have only one operation mode 4 As a matter of fact, inspecting Fig. 6 reveals that the planes normal to $\mathbf{n}_{i}$ and passing through $A_{i}$ do not have a common line of intersection and hence this manipulator cannot exhibit more than one operation mode since condition ii. of Theorem 1 is not satisfied. Figure $7 \mathrm{a}$ shows the projections of planes $\mathscr{P}_{i}$ as lines $\mathscr{L}_{i}$ onto the $X Y$-plane.

95 Nonetheless, the design parameters can be altered so that the condition ii. is satisfied. Changing $n_{i j}, i=$ $1,2, j=1,2,3$ might alter condition i. of Theorem 1, hence $a_{12}, a_{13}$ or $a_{23}$ can be changed so that condition i. is kept intact. From Eq. 22, writing $a_{12}$ as a function of other design parameters and substituting the values form Eq. (41) yields $a_{12}=-\sqrt{3} a$. The design with $a_{12}=-\sqrt{3} a$ is shown in Fig. $7 \mathrm{~b}$ and it exhibits two operation modes. $\tilde{A}_{2}$ represents the initial position of point $A_{2}$. In fact, $a_{13}$ or $a_{23}$ can also be changed similarly to obtain the designs shown in Figs. $7 \mathrm{c}$ and $7 \mathrm{~d}$, respectively. In these figures, $\tilde{A}_{3}$ represents the initial position of point $A_{3}$. Calculating $h_{i}, i=1,2,3$ from Eq. (23) and substituting in the general quadric of Eq. 11] gives the characteristic polynomial of each operation mode as $x_{0}-x_{3}$ and $x_{0}+x_{3}$.

Consequently, it provides an interesting example of how architecture of a manipulator influences its number of operation modes. The procedure explained can be used to design 3-RPS parallel manipulators to have the necessary number of operation modes. Moreover, for a 3-RPS parallel manipulator with two operation

\footnotetext{
${ }^{4}$ When $a=b$, the manipulator at hand can have a constraint singularity when the moving platform is parallel to the fixed base. However, it has only one operation mode. This is because, for a general 3-RPS PM, the variety of its constraint singularity condition is a surface whereas in this case it is reduced to a line parallel to the $Z$-axis and passing through the intersection of the R-joint axes. It is an interesting case of a constraint singularity that does not bifurcate the workspace into multiple operation modes.
} 


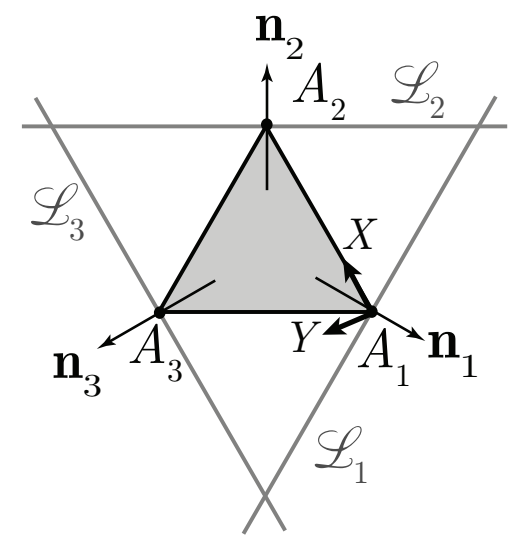

(a) $a_{12}=\sqrt{3} a, \quad a_{13}=\frac{\sqrt{3}}{2} a, \quad a_{23}=\frac{3}{2} a$

One operation mode

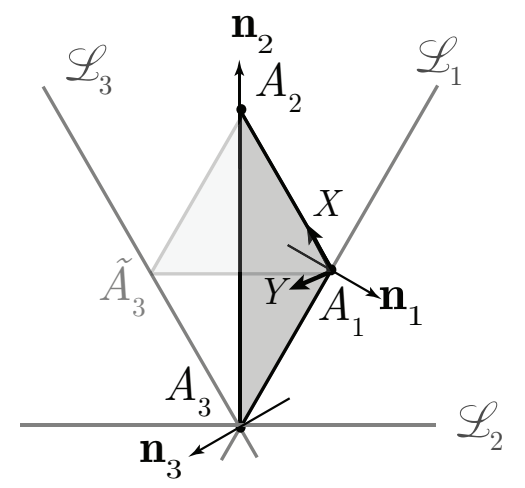

(c) $a_{12}=\sqrt{3} a, \quad a_{13}=-\frac{\sqrt{3}}{2} a, \quad a_{23}=\frac{3}{2} a$

Two operation modes

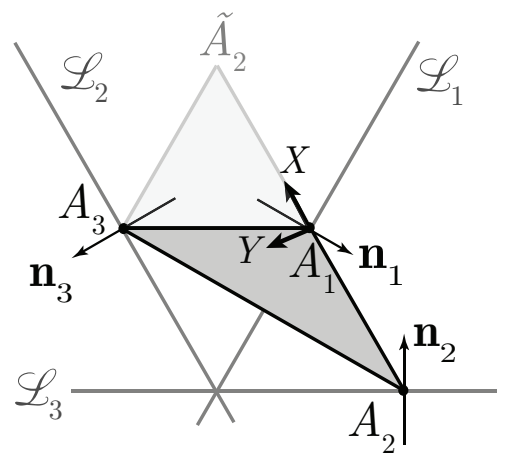

(b) $a_{12}=-\sqrt{3} a, \quad a_{13}=\frac{\sqrt{3}}{2} a, \quad a_{23}=\frac{3}{2} a$

Two operation modes

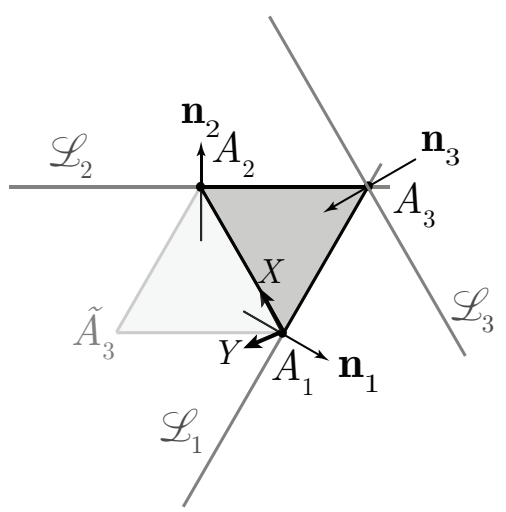

(d) $a_{12}=\sqrt{3} a, \quad a_{13}=\frac{\sqrt{3}}{2} a, \quad a_{23}=-\frac{3}{2} a$

Two operation modes

Figure 7: Slight modification of design parameters can influence the number of operation modes 
modes, the constraint singularity between the operation modes can be escaped by slightly modifying the design parameters such that one of the conditions in Theorem 1 is not fulfilled.

\subsection{Example 3: Arbitrary design parameters}

Finally, a numerical example is studied with the following arbitrary design parameters:

$$
\begin{aligned}
& a_{13}=2, \quad a_{23}=2, \quad b_{23}=3, \\
& n_{11}=-3, \quad n_{21}=5, \quad n_{31}=0, \quad n_{12}=-3, \quad n_{22}=2, \quad n_{32}=0, \quad n_{13}=2, \quad n_{23}=1, \quad n_{33}=0
\end{aligned}
$$

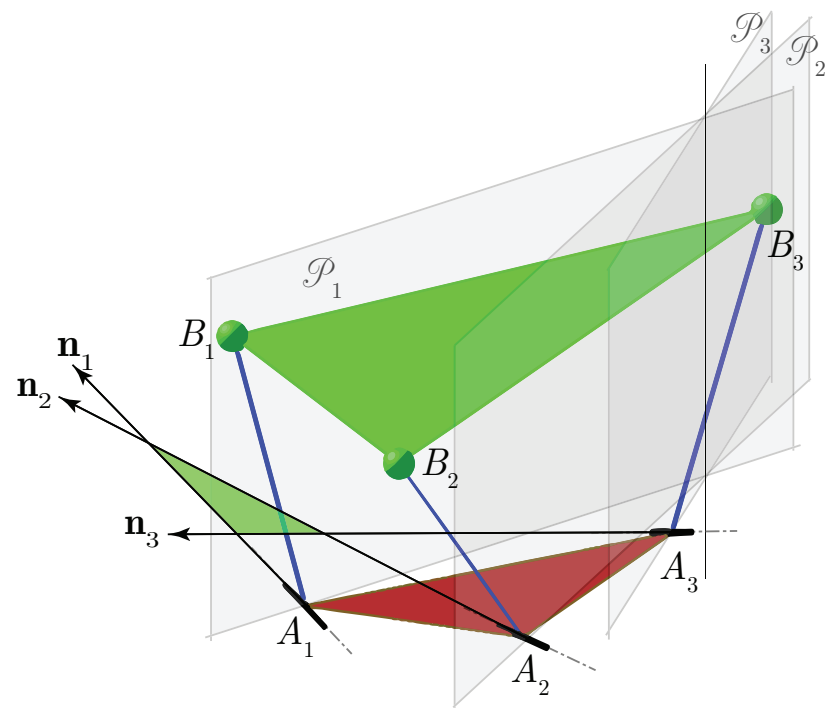

Figure 8: A 3-RPS parallel manipulator with two operation modes characterized by $x_{0}-x_{3}(2+\sqrt{5})=0$ and $x_{0}-x_{3}(2-\sqrt{5})=0$

The remaining design parameters $a_{12}, b_{12}$ and $b_{13}$ are calculated from the relations in Eqs. (22) and (21) so that the manipulator has two operation modes. Thus, $a_{12}=\frac{18}{13}, b_{12}= \pm \frac{135}{91}$ and $b_{13}= \pm \frac{12}{7}$. Fig. 8 shows the architecture of the manipulator at hand, where it can be pointed out that condition i. and ii. of Theorem 1 are satisified. Substituting the design parameters with $b_{12}=-\frac{135}{91}$ and $b_{13}=-\frac{12}{7}$ in Eq. (16) gives

$$
g:=\left(x_{0}-x_{3}(2+\sqrt{5})\right)\left(x_{0}-x_{3}(2-\sqrt{5})\right)=0
$$

whereas, substituting the design parameters with $b_{12}=\frac{135}{91}$ and $b_{13}=\frac{12}{7}$ in Eq. 16. gives

$$
g:=\left(x_{1}-x_{2}(2+\sqrt{5})\right)\left(x_{1}-x_{2}(2-\sqrt{5})\right)=0
$$


The two polynomials $x_{0}-x_{3}(2+\sqrt{5})$ and $x_{0}-x_{3}(2-\sqrt{5})$ or $x_{1}-x_{2}(2+\sqrt{5})$ and $x_{1}-x_{2}(2-\sqrt{5})$ represent the two operation modes of the mechanism 5

Assuming the prismatic joints are actuated, the direct kinematics of the manipulator can be solved by substituting arbitrary values to joint parameters $r_{1}=2, r_{2}=2$ and $r_{3}=3$. The constraint equations $g_{1}=0$ to $g_{6}=0$ and $S_{6}^{2}=0$ can be written from Eqs. (6) and (9). Adding a normalization equation $x_{0}^{2}+x_{1}^{2}+x_{2}^{2}+x_{3}^{2}=1$ (so that $\Delta \neq 0$ in Eq. (5)) yields a set of eight equations to be solved for eight Study parameters. Finding the Groebner basis of the ideal of constraint polynomials with a pure lexicographic monomial ordering $x_{0}<_{\text {lex }} x_{1}<_{\text {lex }} x_{2}<_{\text {lex }} x_{3}<_{\text {lex }} y_{0}<_{\text {lex }} y_{1}<_{\text {lex }} y_{2}<_{\text {lex }} y_{3}$ results in a 16 degree univariate polynomial in variable $y_{3}$. As anticipated, the polynomial can be factorized into two polynomials of degree 8 each corresponding to the two operation modes. It shows that a 3-RPS parallel manipulator can have at most 8 solutions to its direct kinematics in each operation mode. When the joint parameters are fixed, the direct kinematics of a 3-RPS parallel manipulator amounts to locating three points on three fixed circles with centers $A_{i}$ and radii $r_{i}$. To this end, a corollary follows as a consequence of Theorem 1

Corollary 2 For the 3-points on 3-circles problem, if the geometry satisfies the following conditions

i normals to the planes containing the circles and passing through their centers are coplanar,

ii planes containing the circles have a common line of intersection and

iii the triangle formed by the three points is homothetic to the triangle enlosed by three normals to the planes passing through the centers of the circles,

then the 16 degree univariate characteristic polynomial factorizes into two 8 degree polynomials.

For the above-mentioned example, eight real solutions to its direct kinematics problem are found. The solutions form four pairs of manipulator postures, one being the mirror image of another about the $X Y$ plane. Four of these solutions are displayed in Fig. 9

The first two solutions satisfy $\frac{x_{0}}{x_{3}}=2-\sqrt{5}$ or $\frac{x_{1}}{x_{2}}=2+\sqrt{5}$ and hence belong to the operation mode corresponding to $x_{0}-x_{3}(2-\sqrt{5})=0$ or $x_{1}-x_{2}(2+\sqrt{5})=0$, respectively while the last two satisfy $135 \frac{x_{0}}{x_{3}}=2+\sqrt{5}$ or $\frac{x_{1}}{x_{2}}=2-\sqrt{5}$ and hence belong to the operation mode characterized by $x_{0}-x_{3}(2+\sqrt{5})=0$ or $x_{1}-x_{2}(2-\sqrt{5})=0$.

\section{Conclusions and future work}

The influence of design parameters on the number of operation modes of a 3-RPS parallel manipulator with coplanar revolute joints was studied in this paper. The constraint equations of a general 3-RPS

\footnotetext{
${ }^{5}$ To be able to factorize the polynomial $g$, the field of rational numbers must be extended to include $\sqrt{n_{23}^{2}+n_{13}^{2}}$ i.e. $\sqrt{5}$ for this example
} 


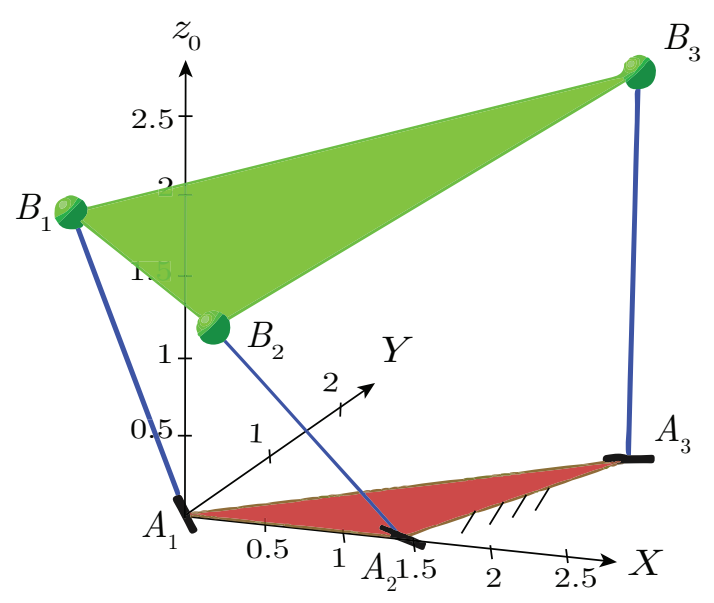

(a) $x_{0}=0.0593, \quad x_{1}=-0.2925, \quad x_{2}=$ $-0.9206, \quad x_{3}=-0.2515, \quad y_{0}=0.0001, \quad y_{1}=$ $-0.8954, y_{2}=0.3571, y_{3}=-0.2658$ OR $x_{0}=.9206, x_{1}=.2515, x_{2}=0.0593, x_{3}=$ $-.2925, \quad y_{0}=-.3571, \quad y_{1}=.2658, \quad y_{2}=$ $0.0001, y_{3}=-.8954$.

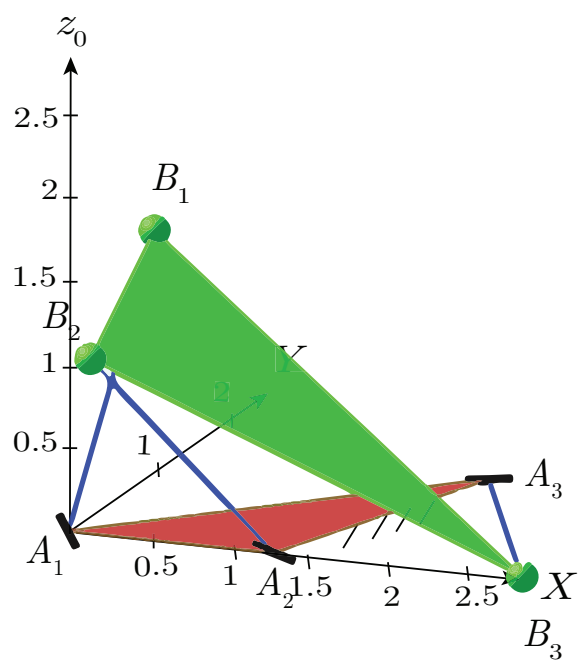

(c) $x_{0}=0.2482, \quad x_{1}=-0.6214, \quad x_{2}=$ $-0.7407, \quad x_{3}=0.0586, \quad y_{0}=-0.2019, \quad y_{1}=$ $-0.7792, y_{2}=0.5742, y_{3}=-0.1490 \mathrm{OR}$ $x_{0}=.7407, x_{1}=-0.0586, x_{2}=.2482, x_{3}=$ $-.6214, \quad y_{0}=-.5742, \quad y_{1}=.1490, \quad y_{2}=$ $-.2019, y_{3}=-.7792$.

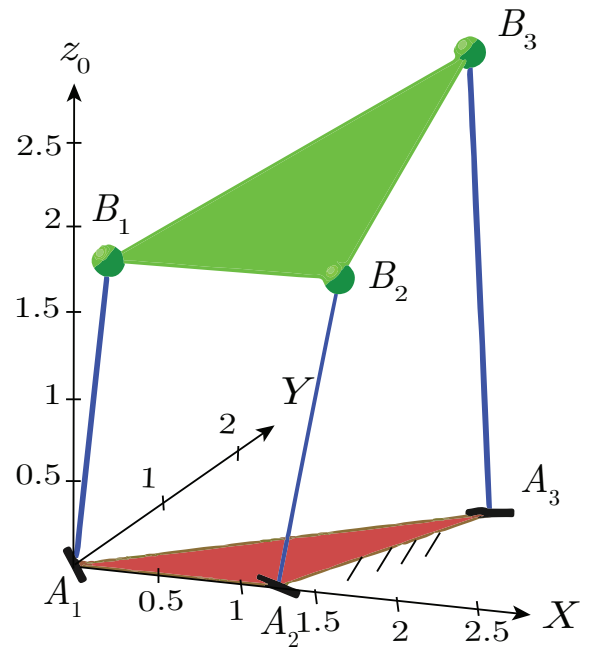

(b) $x_{0}=0.0264, \quad x_{1}=0.0940, \quad x_{2}=$ $-0.9889, x_{3}=-0.1119, \quad y_{0}=-0.1607, y_{1}=$ $-0.9782, y_{2}=-0.1060, y_{3}=0.0775$ OR $x_{0}=-.9889, x_{1}=-.1119, x_{2}=-0.0264, x_{3}=$ $-0.0940, \quad y_{0}=-.1060, \quad y_{1}=0.0775, \quad y_{2}=$ $.1607, y_{3}=.9782$.

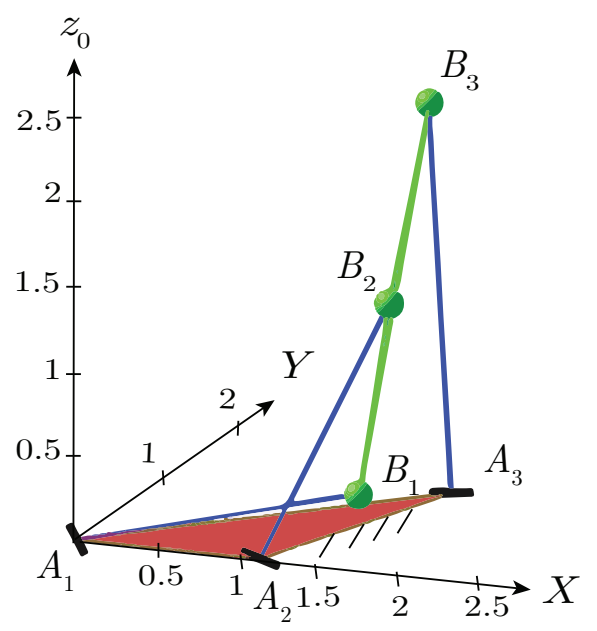

(d) $x_{0}=-0.6304, \quad x_{1}=0.0743, \quad x_{2}=$ $-0.7582, x_{3}=-0.1488, \quad y_{0}=-0.3408, \quad y_{1}=$ 0.5290, $y_{2}=0.1872, y_{3}=0.7541 \mathrm{OR}$ $x_{0}=.7582, x_{1}=.1488, x_{2}=-.6304, x_{3}=$ $0.0743, \quad y_{0}=-.1872, \quad y_{1}=-.7541, \quad y_{2}=$ $-.3408, y_{3}=.5290$.

Figure 9: Solutions to direct kinematics of a 3-RPS manipulator with arbitrary design parameters 
parallel manipulator were derived. The linear combination of plane constraint polynomials were equated to a general quadric in $\mathbb{P}^{7}$ and the coefficients were solved to obtain two solutions with some relations between design parameters. These relations were substituted back into the general quadric and it factorized into two polynomials characterizing two operation modes. The conditions on the design parameters for the existence of two operation modes in 3-RPS manipulator with coplanar revolute joints was summarized as a theorem with proof. The first condition is the homothety between the moving platform triangle and the triangle enclosed by revolute joint axes, while the second condition is when three planes on which the spherical joints are confined to move have a common line of intersection. Two special cases were considered: one that has two operation modes and the other one with one operation mode. For the latter manipulator, it was shown that one can modify the design parameters to be able to have two operation modes. Finally, a numerical example was considered following the proposed theorem. Its characteristic 16 degree univariate polynomial is derived to show that it splits into two polynomials of degree 8 each, representing two operation modes. The direct kinematic solutions lying in each operation mode were shown.

Future work will include the influence of architecture on the operation modes of a 3-RPS parallel manipulator with non-coplanar revolute joints. Additionally, the proposed methodology will be extended to different parallel manipulators.

\section{References}

[1] J. Schadlbauer, D. Walter, M. Husty, The 3-RPS parallel manipulator from an algebraic viewpoint, Mechanism and Machine Theory 75 (Supplement C) (2014) 161 - 176. doi:https://doi.org/10.1016/j.mechmachtheory.2013.12.007. URL http://www.sciencedirect.com/science/article/pii/S0094114X13002504

[2] L. Nurahmi, J. Schadlbauer, M. Husty, P. Wenger, S. Caro, Kinematic analysis of the 3-RPS cube parallel manipulator. in: 38th Mechanisms and Robotics Conference, Vol. 5B, 2014. doi:10.1115/DETC2014-35488 URL http://dx.doi.org/10.1115/DETC2014-35488

[3] K. H. Hunt, Structural kinematics of in-parallel-actuated robot-arms 105 (4) (1983) 705-712. doi:10.1115/1.3258540. URL http://dx.doi.org/10.1115/1.3258540

[4] Z. Huang, Y. Fang, Motion characteristics and rotational axis analysis of three dof parallel robot mechanisms, in: IEEE International Conference on Systems, Man and Cybernetics. Intelligent Systems for the 21st Century, Vancouver, BC, Canada, Oct. 22-25, 1995, pp. 67-71. doi:10.123.

[5] P. Nanua, K. J. Waldron, V. Murthy, Direct kinematic solution of a stewart platform, IEEE Transactions on Robotics and Automation 6 (4) (1990) 438-444. doi:10.1109/70.59354

[6] L.-W. Tsai, Robot Analysis, John Wiley and Sons, Inc., 1999.

[7] J. Schadlbauer, M. Husty, S. Caro, P. Wenger, Self-motions of 3-RPS manipulators, Frontiers of Mechanical Engineering 8(1) (2013) 62-69.

[8] D. Chablat, R. Jha, F. Rouillier, G. Moroz, Workspace and joint space analysis of the 3-RPS parallel robot, in: ASME 2013 International Design Engineering Technical Conferences and Computers and Information in Engineering Conference, Vol. 5A, 2014, pp. 1-10.

[9] L. Nurahmi, S. Caro, P. Wenger, Design of 3-RPS parallel manipulators based on operation modes, in: Proceedings of the 14th IFTOMM World Congress, October 25-30, 2015, Taipei, Taiwan, 2015. doi:10.6567/IFToMM.14TH.WC.0S2.040 
[10] J. Schadlbauer, D. Walter, M. Husty, A complete analysis of the 3-RPS parallel manipulator, in: Machines and Mechanisms, Narosa Publishing House, New Delhi, India, 2012, pp. 410-419.

[11] J. Gallardo, H. Orozco, J. M. Rico, Kinematics of 3-RPS parallel manipulators by means of screw theory The International Journal of Advanced Manufacturing Technology 36 (5) (2008) 598-605. doi:10.1007/s00170-006-0851-5. URL https://doi.org/10.1007/s00170-006-0851-5

[12] J. Carretero, M. Nahon, C. Gosselin, B. Buckham, Kinematic analysis of a three-dof parallel mechanism for telescope applications, in: Proceedings of the 1997 ASME Design Engineering Technical Conferences, 1997.

[13] A. Hernández, O. Altuzarra, C. Pinto, E. Amezua, Transitions in the velocity pattern of lower mobility parallel manipulators, Mechanism and Machine Theory 43 (2008) 738-753.

[14] D. Verde, S. D. Stan, M. Manic, R. Balan, V. Matie, Kinematics analysis, workspace, design and control of 3-RPS and triglide medical parallel robots, in: 2009 2nd Conference on Human System Interactions, 2009, pp. 103-108. doi: 10.1109/HSI.2009.5090962

[15] M. L. Husty, H. P. Schröcker, 21st Century Kinematics, Springer-Verlag London, London, 2013, Ch. Kinematics and Algebraic Geometry, pp. 85-123.

[16] D. A. Cox, J. Little, D. O'Shea, Ideals, Varieties, and Algorithms: An Introduction to Computational Algebraic Geometry and Commutative Algebra, 3/e (Undergraduate Texts in Mathematics), Springer-Verlag New York, Inc., Secaucus, NJ, USA, 2007.

[17] D. Zlatanov, I. A. Bonev, C. M. Gosselin, Constraint singularities of parallel mechanisms, in: 2002 IEEE International Conference on Robotics and Automation (ICRA'02), Vol. 1, IEEE, 2002, pp. 496-502.

[18] S. Amine, O. Mokhiamar, S. Caro, Classification of 3T1R parallel manipulators based on their wrench graph, Journal of Mechanisms and Robotics 9 (1) (2016) 011003-011003-10. 